\title{
DYNAMICAL PREDICTION TECHNIQUE FOR GEOSIMULATION USING MULTISPECTRAL REMOTE SENSING DATA
}

\author{
I. E. Villalon-Turrubiates ${ }^{1}$ \\ ${ }^{1}$ Instituto Tecnológico y de Estudios Superiores de Occidente (ITESO), Universidad Jesuita de Guadalajara \\ Periférico Sur Manuel Gómez Morín 8585, 45604 Tlaquepaque Jalisco México - villalon@iteso.mx
}

Commission IV, ICWG III/IVb and WG IV/4

KEY WORDS: Dynamical Analysis, Prediction Model, Remote Sensing, Image Processing, Image Analysis, Multispectral Analysis.

\begin{abstract}
:
The analysis of dynamical models for prediction and geosimulation using the information extracted from a geographical region processed from the data provided by multispectral remote sensing systems provides useful information for urban planning and resource management. However, several topics of interest on this particular matter are still to be properly studied. Using the remote sensing data that has been extracted from multispectral images from a particular geographic region in discrete time, its dynamic study is performed in both, spatial resolution and time evolution, in order to obtain the dynamical model of the physical variables and the evolutionary information about the data. This provides a background for understanding the future trends in development of the dynamics inherent in the multispectral and high-resolution images. This proposition is performed via an intelligent computational paradigm based on the use of dynamical filtering techniques modified to enhance the quality of reconstruction of the data extracted from multispectral remote sensing images and using high-performance computational techniques to unify the available data scheme with its dynamic analysis and, therefore, provide a behavioral model of the sensed data.
\end{abstract}

\section{INTRODUCTION}

Dynamic is a term that refers to a phenomenon that produces time changing patterns, the characteristics of that pattern at a particular time is related with those at other times.

The term is nearly synonymous with time evolution or pattern of change (Luenberger, 1979). Nearly all the observed phenomena every day, or in a scientific research, have important dynamic aspects. Scientific examples may arise in:

1. A physical system, such as a signal traveling through the space, a home heating system, or in the mining of a mineral deposit.

2. A social system, such as the movement within an organization hierarchy, the evolution of a tribal class system, or the behavior of an economic structure.

3. A life system, such as that of genetic transference, ecological decay, or population growth.

Many dynamic systems can be understood and analyzed intuitively without resort to mathematics and without development of a general theory of dynamics. However, in order to approach unfamiliar complex situations efficiently, it is necessary to proceed systematically. Mathematics can provide the required economy of language and conceptual framework; therefore, the term dynamics takes a dual meaning. It is a term for the time evolution phenomenon in the real world, and a term for that part of mathematical science that is used for its representation and analysis (Luenberger, 1979). Dynamic systems are represented mathematically in terms of either differential or difference equations. These equations provide the structure for representing time linkages among variables.
The use of either differential or difference equations to represent dynamic behavior corresponds, respectively, to whether the behavior is viewed as occurring in continuous or discrete time.

Continuous time corresponds to the usual conception, where time is regarded as a continuous variable and is often viewed as flowing and smoothly passing (Luenberger, 1979). In mathematical terms, continuous time is quantified in terms of the continuum of real numbers.

Discrete time consists of an ordered sequence of points rather than a continuum. In terms of applications, it is convenient to introduce this type of time when events and consequences either occurring are accounted for only at discrete time periods, such as daily, monthly, or yearly. Accordingly, dynamic behavior viewed in discrete time is usually described by equations relating the value of a variable at one time to the values at adjacent times. Such equations are called difference equations.

\section{DYNAMIC MODEL}

An innovative paradigm that has been developed is presented, its objective is the mathematical analysis of the dynamical model in both, spatial resolution and time evolution, of a particular geographical region obtained from multispectral remote sensing data (MRSD) in discrete time.

This is performed via the Multispectral Dynamic Filtering (MDF) method, which unifies the MRSD mapping scheme with its dynamic analysis to provide a high-resolution mapping of the MRSD in discrete time. If the attributes of interest of a system are changing in time, then it is referred to as a dynamic system. The MDF process provides the mathematical model of change in space resolution and time evolution of such a dynamic system (Grewal et al., 2001). 


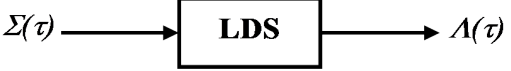

a. CT domain: $\tau$-continuous time argument.

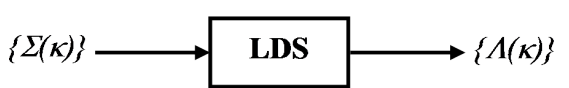

b. DT domain: $\kappa$-discrete time argument.

Figure 1. Representation of linear dynamic systems.

\subsection{Mathematical model in continuous time}

The model of MRSD of interest treated as a linear dynamic system (LDS) is presented in its state variables (Falkovich et al., $1989)$ described over the continuous time (CT) domain $(\tau \in \mathfrak{I})$ and the discrete time (DT) domain $(\kappa \in \Re)$. The LDS is represented in CT and DT as shown in Figures 1(a) and 1(b), respectively.

For the LDS presented in CT and DT, $\sum(\tau)$ and $\left\{\sum(\kappa)\right\}$ are the inputs to the linear system, $\Lambda(\tau)$ and $\{\Lambda(\kappa)\}$ are the outputs of the linear system, respectively.

The model of the equation of observation (EO) in CT is represented as $\Sigma(\tau)=S(\Lambda(\tau))+v(\tau)$ (Falkovich et al., 1989), where $v(\tau)$ is the white observation Gaussian noise and $\tau \in \mathfrak{I}$, starting at $\tau_{0}$ (initial moment of continuous time), and the linear amplitude-modulated model (Falkovich et al., 1989) is $S(\Lambda(\tau))=\Lambda(\tau) S_{0}(\tau)$, where $S_{0}(\tau)$ represents the deterministic carrier of the image frame of a given model, and $\Lambda(\tau)$ is the unknown stochastic information process to be estimated via processing of the image observation data frame $\sum(\tau)$. Is assumed that $\Lambda(\tau)$ satisfies the dynamical model specified by the $N$-th order linear differential equation (Shkvarko et al., 2007)

$$
\begin{aligned}
& \frac{d^{N} \Lambda(\tau)}{d \tau^{N}}+\alpha_{N-1} \frac{d^{N-1} \Lambda(\tau)}{d \tau^{N-1}}+\cdots+\alpha_{0} \Lambda(\tau) \\
& =\beta_{N-1} \frac{d^{N-1} \xi(\tau)}{d \tau^{N-1}}+\cdots+\beta_{0} \xi(\tau)
\end{aligned}
$$

where $\alpha$ and $\beta$ are the constant coefficients of the dynamical system model for evolution of the MRSD $\Lambda(\tau)$.

This stochastic model can be redefined as follows: the differential equation (1) may be transformed into a system of linear differential equations of the first order via performing the following replacement of variables

$$
\begin{gathered}
z_{1}(\tau)=\Lambda(\tau), \\
z_{2}(\tau)=\frac{d z_{1}(\tau)}{d \tau}+\alpha_{N-1} z_{1}(\tau)-\beta_{N-1} \xi(\tau), \\
\vdots \\
z_{N}(\tau)=\frac{d z_{N-1}(\tau)}{d \tau}+\alpha_{1} z_{1}(\tau)-\beta_{1} \xi(\tau), \\
\frac{d z_{N}(\tau)}{d \tau}=-\alpha_{0} z_{1}(\tau)+\beta_{0} \xi(\tau) \\
\mathbf{z}(\tau)=\left(\begin{array}{llll}
\left.z_{1}(\tau) \quad z_{2}(\tau) \quad \ldots \quad z_{N}(\tau)\right)^{T}
\end{array}\right.
\end{gathered}
$$

Based on the replacement of variables specified by (2), the dynamic differential equation model (1) can be now represented in a canonical vector-matrix form as follows

$$
\frac{d \mathbf{z}(\tau)}{d \tau}=\mathbf{F z}(\tau)+\mathrm{G} \xi(\tau), \quad \Lambda(\tau)=\mathrm{Cz}(\tau)
$$

$$
\begin{gathered}
\mathbf{F}=\left[\begin{array}{ccccc}
-\alpha_{N-1} & 1 & 0 & \ldots & 0 \\
-\alpha_{N-2} & 0 & 1 & \ldots & 0 \\
\ldots & \ldots & \ldots & \ldots & \ldots \\
-\alpha_{1} & 0 & 0 & \ldots & 1 \\
-\alpha_{0} & 0 & 0 & \ldots & 0
\end{array}\right], \\
\mathbf{G}=\left[\begin{array}{c}
\beta_{N-1} \\
\beta_{N-2} \\
\ldots \\
0
\end{array}\right], \\
\mathbf{C}=\left[\begin{array}{llll}
1 & 0 & \ldots & 0
\end{array}\right] .
\end{gathered}
$$

The representation in the form of (4) is referred to as a canonical equation of linear dynamic system in state variables in continuous time. Here, $\mathbf{z}(\tau)$ is the state vector, the vector $\mathbf{C}$ defines a linear operator that introduces the relation between the MRSD in continuous time and the state vector $\mathbf{z}(\tau), \mathbf{F}$ is a transition matrix, $\mathbf{G}$ is a transition vector, and $\xi(\tau)$ represents the white model generation noise vector characterized by the statistics, $\langle\xi(\tau)\rangle=\mathbf{0}$ and $\left\langle\xi(\tau) \xi^{T}\left(\tau^{\prime}\right)\right\rangle=\mathbf{P}_{\xi}(\tau) \sigma\left(\tau-\tau^{\prime}\right)$, respectively. Here, $\mathbf{P}_{\xi}(\tau)$ is referred to as state model disperse matrix that characterizes the dynamics of the state variances developed in continuous evolution time $\tau\left(\tau_{0} \rightarrow \tau\right)$ starting from the initial instant $\tau_{0}$.

The dynamic model equation in the continuous time states the relation between the MRSD map $\sum(\tau)$ extracted from the remote sensing scene, thus the desired dynamical map $\Lambda(\tau)$ is (Shkvarko et al., 2007)

$$
\Sigma(\tau)=\mathrm{S}_{0}(\tau) \mathrm{C}(\tau) \mathrm{z}(\tau)+\mathrm{v}(\tau)=\mathrm{H}(\tau) \mathrm{z}(\tau)+\mathrm{v}(\tau),
$$

where $\mathbf{H}(\tau)=\mathbf{S}_{0}(\tau) \mathbf{C}(\tau)$.

The stochastic differential model of equations (4) and (6) allows applying the theory of dynamical filtration to reconstruct the desired MRSD map in continuous time incorporating the a priori model of dynamical information about the MRSD.

The aim of the dynamic filtration is to find an optimal estimate of the desired MRSD, $\widehat{\boldsymbol{\Lambda}}(\tau)=\mathbf{C} \hat{\mathbf{z}}(\tau)$, developed in continuous time $\tau\left(\tau_{0} \rightarrow \tau\right)$ via processing the MRSD maps $\sum(\tau)$ extracted from the remote sensing scenes and taking into considerations the a priori dynamic model of the desired MRSD map specified through the state equation (4).

The optimal dynamic filter when applied to the RSG maps $\sum(\tau)$ specified by the dynamic image model (6) must provide the optimal estimation of the desired MRSD map $\widehat{\Lambda}(\tau)=\mathbf{C} \hat{\mathbf{z}}(\tau)$. 


\subsection{Mathematical model of the MRSD}

The canonical solution to equation (4) in state variables for discrete time $\kappa$ is expressed as follows (Falkovich et al., 1989)

$$
\begin{aligned}
& \mathbf{z}(\kappa+1)=\boldsymbol{\Phi}(\kappa) \mathbf{z}(\kappa)+\Gamma(\kappa) \xi(\kappa), \\
& \mathbf{\Lambda}(\kappa)=\mathbf{C}(\kappa) \mathbf{z}(\kappa),
\end{aligned}
$$

where $\boldsymbol{\Phi}(\kappa)=\mathbf{F}\left(\tau_{\kappa}\right) \Delta \tau+\mathbf{I}, \boldsymbol{\Gamma}(\kappa)=\mathbf{G}\left(\tau_{\kappa}\right) \Delta \tau$ and $\Delta \tau$ represents the continuous time sampling interval for dynamical modelling of the MRSD map in discrete time. The statistical characteristics of the a priori information are as follows.

Generating model noise $\{\xi(\kappa)\}$ :

$$
\langle\xi(\kappa)\rangle=\mathbf{0},\left\langle\xi(\kappa) \xi^{T}\left(\kappa^{\prime}\right)\right\rangle=\mathbf{P}_{\xi}\left(\kappa, \kappa^{\prime}\right)
$$

Observation (MRSD) noise $\{v(\kappa)\}$ :

$$
\langle\mathbf{v}(\kappa)\rangle=\mathbf{0},\left\langle\mathbf{v}(\kappa) \mathbf{v}^{T}\left(\kappa^{\prime}\right)\right\rangle=\mathbf{P}_{v}\left(\kappa, \kappa^{\prime}\right) .
$$

State vector $\{\zeta(\kappa)\}$ :

$$
\langle\mathbf{z}(0)\rangle=\mathbf{m}_{z}(\mathbf{0}),\left\langle\mathbf{z}(0) \mathbf{z}^{T}(0)\right\rangle=\mathbf{P}_{z}(0) .
$$

where 0 argument implies the initial state for initial time instant $(\kappa=0)$. The disperse matrix $\mathrm{P}_{z}(0)$ satisfies the following disperse dynamic equation (Shkvarko et al., 2007)

$$
\begin{aligned}
\mathbf{P}_{\mathrm{z}}(\kappa+1)=\langle\mathbf{z}(\kappa+1) & \left.\mathbf{z}^{T}(\kappa+1)\right\rangle \\
& =\boldsymbol{\Phi}(\kappa) \mathbf{P}_{\mathrm{z}}(\kappa) \boldsymbol{\Phi}^{T}(\kappa) \\
& +\boldsymbol{\Gamma}(\kappa) \mathbf{P}_{\xi}(\kappa) \boldsymbol{\Gamma}^{T}(\kappa) .
\end{aligned}
$$

\subsection{Optimal and dynamic MRSD technique}

The strategy is to design an optimal decision procedure that, when applied to all MRSD observations will provide an optimal solution to the state vector $\mathbf{z}(\kappa)$ subjected to its prior defined dynamic model given by the stochastic dynamic equation (7).

The estimate of the state vector optimally defined in the sense of the Bayesian minimum risk strategy (Shkvarko, 2004a) in discrete time $\kappa$ can be represented in the conditioned form

$$
\underset{\text { opt }}{\hat{\mathbf{z}}(\kappa)}=\langle\mathbf{z}(\kappa) \mid \Sigma(0), \Sigma(1), \ldots, \Sigma(\kappa)\rangle,
$$

were $\langle\cdot\rangle$ represents an ensemble averaging operator.

For discrete time, the design procedure is based on the concept of mathematical induction (Falkovich et al., 1989). This is a supposition that after $\kappa$ observations $\{\Sigma(0), \Sigma(1), \ldots, \Sigma(\kappa)\}$ the optimal estimate is

$$
\hat{\mathbf{z}}(\kappa)=\underset{\text { opt }}{\hat{\mathbf{z}}(\kappa)}
$$

In order to use the estimate $\hat{\mathbf{z}}(\kappa)$ it is necessary to design an algorithm that produces the optimal estimate $\mathbf{z}(\kappa+1)$ incorporating new measurements $\Sigma(\kappa+1)$ according to the state dynamic equation (7). This is, we have to design an optimal decision procedure (optimal filter) that, when applied to all reconstructed MRSD maps $\{\boldsymbol{\Sigma}(\kappa)\}$ ordered in discrete time $\kappa\left(\kappa_{0} \rightarrow \kappa\right)$, provides an optimal reconstruction of the desired MRSD map represented via the estimate of the state vector $\mathbf{z}(\kappa)$ subject to the numerical dynamic model (7). To proceed with derivation of such a filter, the state dynamic equation (7) in discrete time $\kappa$ is

$$
\mathbf{z}(\kappa+1)=\boldsymbol{\Phi}(\kappa) \mathbf{z}(\kappa)+\Gamma(\kappa) \xi(\kappa) .
$$

\subsection{Dynamic MRSD reconstruction}

According to the dynamical model of equation (14), the anticipated mean value for the state vector can be expressed as

$$
\mathbf{m}_{\mathbf{z}}(\kappa+1)=\langle\mathbf{z}(\kappa+1)\rangle=\langle\mathbf{z}(\kappa+1) \mid \hat{\mathbf{z}}(\kappa)\rangle .
$$

The $\mathbf{m}_{\mathrm{z}}(\kappa+1)$ is considered as the a priori conditional mean value of the state vector for the $(\kappa+1)$-st estimation step, thus, from equations (14) and (15)

$$
\begin{gathered}
\mathbf{m}_{\mathrm{z}}(\kappa+1)=\boldsymbol{\Phi}\langle\mathbf{z}(\kappa) \mid \boldsymbol{\Sigma}(0), \boldsymbol{\Sigma}(1), \ldots, \boldsymbol{\Sigma}(\kappa)\rangle+\boldsymbol{\Gamma}\langle\boldsymbol{\xi}(\kappa)\rangle \\
=\boldsymbol{\Phi} \hat{\mathbf{z}}(\kappa),
\end{gathered}
$$

hence, the prognosis of the mean value becomes

$$
\mathbf{m}_{\mathrm{z}}(\kappa+1)=\boldsymbol{\Phi} \hat{\mathbf{z}}(\kappa) .
$$

From the analysis of equations (14) thru (17), it is possible to deduce that given the fact that the particular MRSD map $\boldsymbol{\Sigma}(\kappa)$ is treated at discrete time $\kappa$, it makes the previous reconstructions $\{\boldsymbol{\Sigma}(0), \boldsymbol{\Sigma}(1), \ldots, \boldsymbol{\Sigma}(\kappa)\}$ irrelevant; hence, the optimal filtering strategy is reduced to the dynamical one step predictor described by the equation (14).

Using these derivations, the dynamical estimation strategy can be modified to the one step optimal prediction procedure (Shkvarko et al., 2007)

$$
\begin{aligned}
& \hat{\mathbf{z}}(\kappa+1) \\
& =\langle\mathbf{z}(\kappa+1) \mid \boldsymbol{\Sigma}(0), \boldsymbol{\Sigma}(1), \ldots, \boldsymbol{\Sigma}(\kappa), \boldsymbol{\Sigma}(\kappa+1)\rangle ; \\
& \quad \hat{\mathbf{z}}(\kappa+1)=\langle\mathbf{z}(\kappa+1) \mid \hat{\mathbf{z}}(\kappa) ; \boldsymbol{\Sigma}(\kappa+1)\rangle ; \\
& \quad \hat{\mathbf{z}}(\kappa+1)=\left\langle\mathbf{z}(\kappa+1) \mid \boldsymbol{\Sigma}(\kappa+1) ; \mathbf{m}_{\mathrm{z}}(\kappa+1)\right\rangle .
\end{aligned}
$$

For the current $(\kappa+1)-s t$ discrete evolution time estimation/prediction step, the dynamical MRSD map estimate of the equation (6) in discrete time becomes

$$
\boldsymbol{\Sigma}(\kappa+1)=\mathbf{H}(\kappa+1) \mathbf{z}(\kappa+1)+\mathbf{v}(\kappa+1),
$$

with the a priori predicted mean calculated by the equation (15) for the desired state vector given by (14).

Applying the Wiener minimum risk strategy (Shkvarko, 2004b) to solve the equation (19) with respect to the state vector $\mathbf{z}(\kappa)$ and taking into account the a priori information summarized by the equations (8) thru (10), the dynamic solution for the MRSD map state vector is obtained, defined by the Equation (20) as

$$
\begin{aligned}
\hat{\mathbf{z}}(\kappa+1)=\mathbf{m}_{\mathrm{z}}(\kappa+1) & \\
& +\boldsymbol{\Theta}(\kappa+1)[\mathbf{\Sigma}(\kappa+1) \\
& \left.-\mathbf{H}(\kappa+1) \mathbf{m}_{\mathrm{z}}(\kappa+1)\right]
\end{aligned}
$$

where the desired dynamic filter operator is defined as

$$
\begin{gathered}
\boldsymbol{\Theta}(\kappa+1)=\mathbf{K}_{\Theta}(\kappa+1) \mathbf{H}^{T}(\kappa+1) \mathbf{P}_{v}^{-1}(\kappa+1), \\
\mathbf{K}_{\Theta}(\kappa+1)=\left[\boldsymbol{\Psi}_{\Theta}(\kappa+1)+\mathbf{P}_{Z}^{-1}(\kappa+1)\right]^{-1}, \\
\boldsymbol{\Psi}_{\Theta}(\kappa+1)=\mathbf{H}^{T}(\kappa+1) \mathbf{P}_{v}^{-1}(\kappa+1) \mathbf{H}(\kappa+1) .
\end{gathered}
$$


Using the derived filter equations (20) thru (23) and the initial MRSD map state model of equation (7), the optimal filtering procedure for the dynamic reconstruction of the desired MRSD map can be represented in discrete time $\kappa$ as Equation (24)

$$
\begin{aligned}
\widehat{\Lambda}(\kappa+1)=\boldsymbol{\Phi}(\kappa) & \hat{\mathbf{z}}(\kappa) \\
& +\boldsymbol{\Theta}(\kappa+1)[\boldsymbol{\Sigma}(\kappa+1) \\
& -\mathbf{H}(\kappa+1) \boldsymbol{\Phi}(\kappa) \hat{\mathbf{z}}(\kappa)]
\end{aligned}
$$

\section{MULTISPECTRAL DYNAMIC FILTER}

The described technique provides the dynamic MRSD reconstruction based on a collection of MRSD maps extracted from remote sensing scenes (Perry et al., 2002).

This developed methodology is referred to as the Multispectral Dynamic Filter (MDF) method. The MDF method is defined in the form of equation (24) and based on the collection of MRSD maps in discrete time as Equation (25)

$$
\begin{aligned}
\widehat{\boldsymbol{\Lambda}}_{M D F}=\widehat{\Lambda}(\kappa+1) & \\
\widehat{\Lambda}_{M D F}=\boldsymbol{\Phi}(\kappa) \hat{\mathbf{z}}(\kappa) & \\
& +\boldsymbol{\Theta}(\kappa+1)[\boldsymbol{\Sigma}(\kappa+1) \\
& -\mathbf{H}(\kappa+1) \boldsymbol{\Phi}(\kappa) \hat{\mathbf{z}}(\kappa)]
\end{aligned}
$$

Here, the observation vector $\boldsymbol{\Sigma}$ is formed by the threshold values of the same $(i, j)$-th pixel through the different MRSD maps of the atlas collection in the discrete time $\kappa$.

The estimate vector $\mathbf{z}$ is formed by the estimation values $\Lambda$ one step prior in the same current discrete time. Figure 2 shows the detailed computational structure of the MDF method for environmental MRSD reconstruction of remote sensing imagery.

\section{SIMULATION EXPERIMENT}

Remote sensing is a tool or technique similar to mathematics (Mather, 2004) that use sensors to measure the amount of electromagnetic radiation (EMR) existing an object or geographic area from a distance and extracts valuable information from the data using mathematically and statistically based algorithms (Jensen, 2005). It functions in harmony with other spatial data collections techniques or tools of the mapping sciences, including geographic information systems (GIS) and is used for geosimulation processes (Fussell et al., 1986).

In the simulation results, a set of 40 MRSD maps were extracted from multispectral remote sensing images with high-resolution values for of spectral and spatial resolution and for a particular geographical scene. The MDF methodology is applied to the collection of MRSD maps (Villalon, 2014) based on the computational structure described in Figure 2.

First, the collection of MRSD maps (Marple, 1987) extracted in different times (discrete) for the same scene is set for the simulation. Therefore, the discrete evolution time $\kappa$ equals to 40 . Second, the pixel evolution vector $\boldsymbol{\Sigma}_{i j}$ is defined for this simulation as

$$
\boldsymbol{\Sigma}_{i j}=\left(\begin{array}{llll}
\widehat{\boldsymbol{\Sigma}}_{i j, 1} & \widehat{\boldsymbol{\Sigma}}_{i j, 2} & \ldots & \widehat{\boldsymbol{\Sigma}}_{i j, 40}
\end{array}\right),
$$

where $\widehat{\boldsymbol{\Sigma}}$ represents the threshold values of the same $(i, j)$-th pixel from the MRSD maps (Yli-Harja et al., 1991).
This is the observation signal to be post-processed with the dynamic post-processing method. Third, the measurement matrix $\mathbf{H}$ and the state transition matrix $\boldsymbol{\Phi}$ are simplified to $\mathbf{I}$ because the equation of observation (7) and the stochastic dynamic state equation (19) are supposed to be ideal (noiseless, because the observation vector is directly extracted from the MRSD maps).

The dynamic filter operator (gain matrix) $\Theta$ determines the variance evolution of the observation values (26) of the dynamically reconstructed MRSD. The initial conditions are the initial observation value $\boldsymbol{\Sigma}(0)$ and its initial estimation $\widehat{\Lambda}(0)=$ $\boldsymbol{\Lambda}\{\boldsymbol{\Sigma}(0)\}$.

The MDF method specified by equation (25) is applied to estimate the ultimate value $\widehat{\boldsymbol{\Lambda}}$ that is the next $(\kappa+1)-s t$ continuous time step of the observation vector $\boldsymbol{\Sigma}_{i j}$. This process is performed through all the $\{(i, j)\}$ pixels of the MRSD maps to obtain a single aggregated MRSD map $\widehat{\Lambda}_{M D F}$.

The simulation results of application of the developed MDF method are presented in Figure 3. Figures 3(a) thru 3(e) show the first five MRSD maps (1024x1024-pixels) extracted from the first five remote sensing scenes that corresponds to the metropolitan area of the city of Guadalajara, in Mexico. This is performed in different time $(\kappa=1,2,3,4$ and 5) for the time evolution analysis, respectively.

Figure 3(f) shows the dynamic MRSD map reconstructed with the application of the MDF method for the $(\kappa+1)$ time step $(\kappa=41)$ specified by the computational structure described in Figure 2.

The MRSD map were reconstructed in a discrete time $\kappa$, therefore, the MDF method produces the desired dynamic MRSD prediction for the next discrete time step $(\kappa+1)$, which represents the prediction of changes in spatial resolution and time evolution (Johannsen et al., 2003).

\section{COMPUTATIONAL ALGORITHM}

The detailed stages of the computational algorithm for the MDF methodology is summarized as follows

1. Set a collection of discrete time $(\kappa)$ MRSD maps extracted from the remote sensing imagery for a particular scene.

2. For each $(i, j)$-th pixel on the MRSD maps, perform the following process:

- $\quad$ Set the pixel-based evolution vector $\boldsymbol{\Sigma}_{i j}$, which contains the threshold MRSD values for the pixel in discrete time $\kappa$.

- $\quad$ Apply the MDF method to the vector $\boldsymbol{\Sigma}_{i j}$ to obtain the prediction $\widehat{\Lambda}(\kappa+1)$, which conform the matrix $\widehat{\Lambda}_{i j}$.

3. The reconstructed $\widehat{\boldsymbol{\Lambda}}_{i j}$ matrix conform the $\widehat{\boldsymbol{\Lambda}}_{M D F}$ dynamic MRSD image in discrete time $\kappa$. 


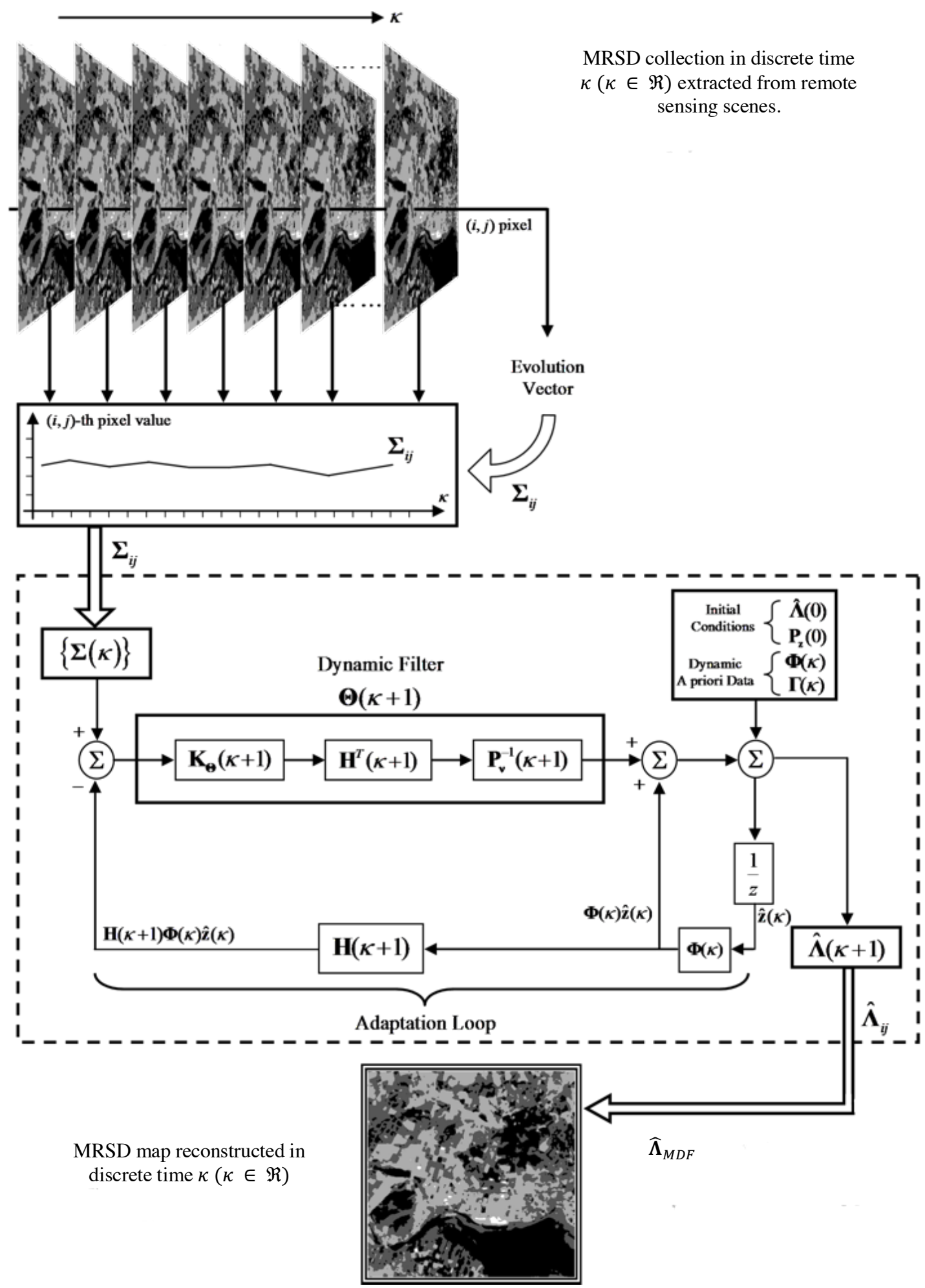

Figure 2. Computational structure of the MDF method. 


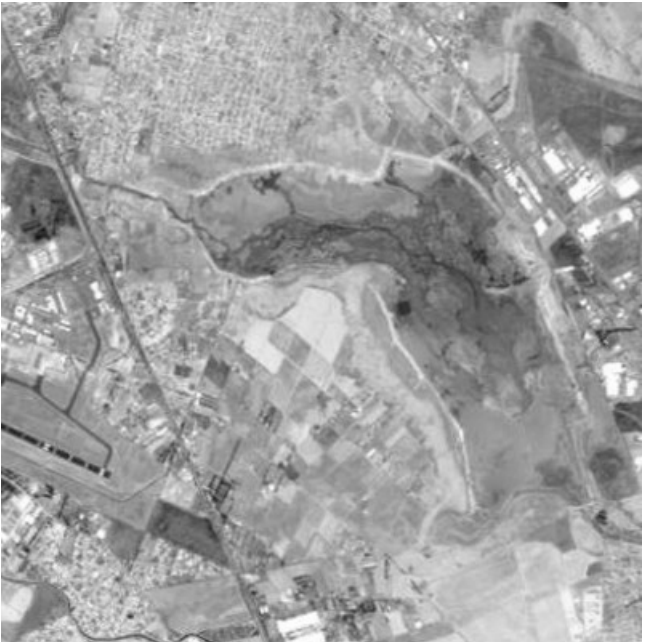

a. MRSD map from the RS scene for $\kappa=1$ discrete time.

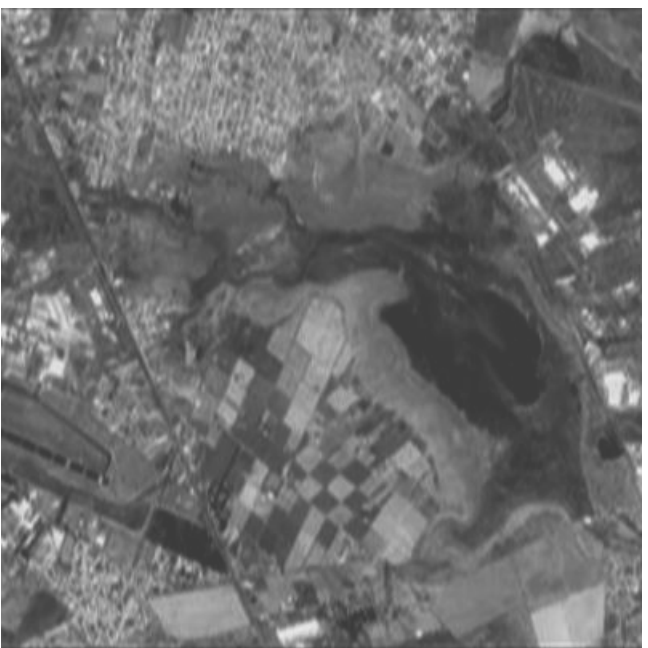

c. MRSD map from the remote sensing scene for $\kappa=3$ discrete time.

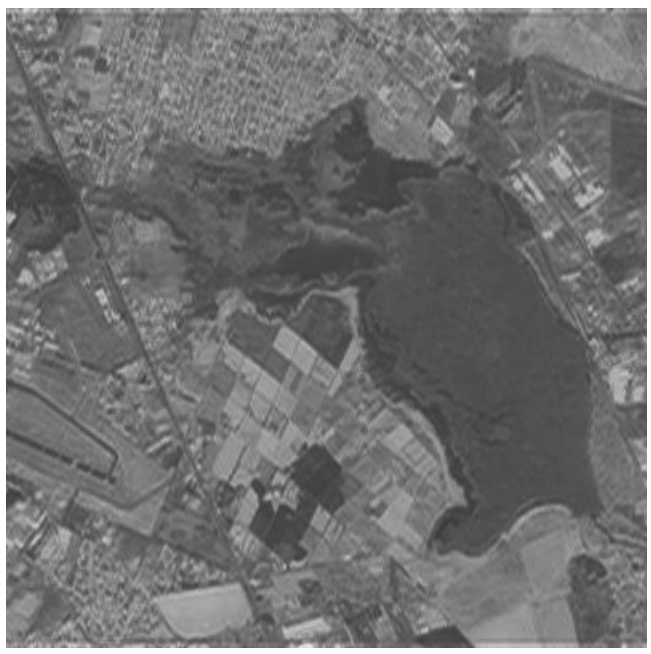

e. MRSD map from the remote sensing scene for $\kappa=5$ discrete time.

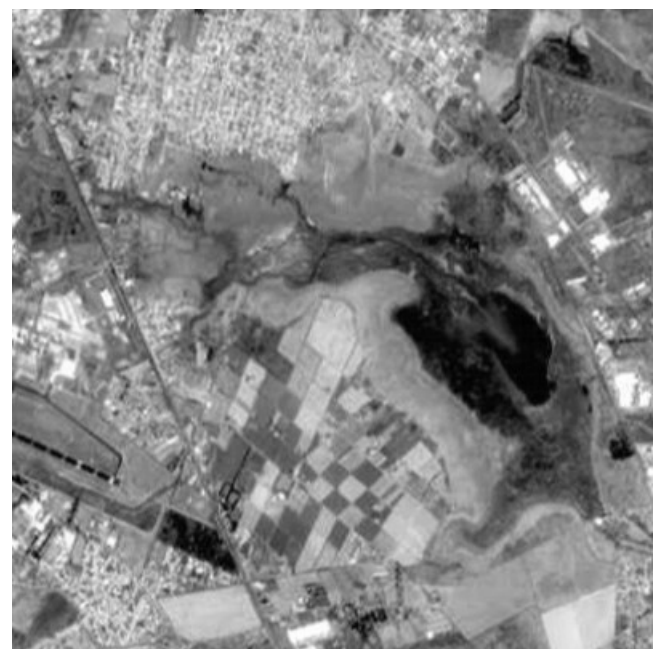

b. MRSD map from the remote sensing scene for $\kappa=2$ discrete time.

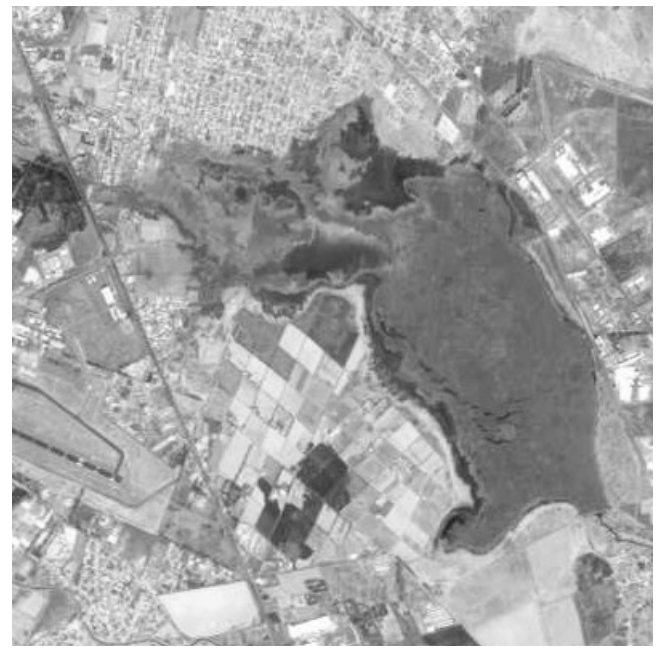

d. MRSD map from the remote sensing scene for $\kappa=4$ discrete time.

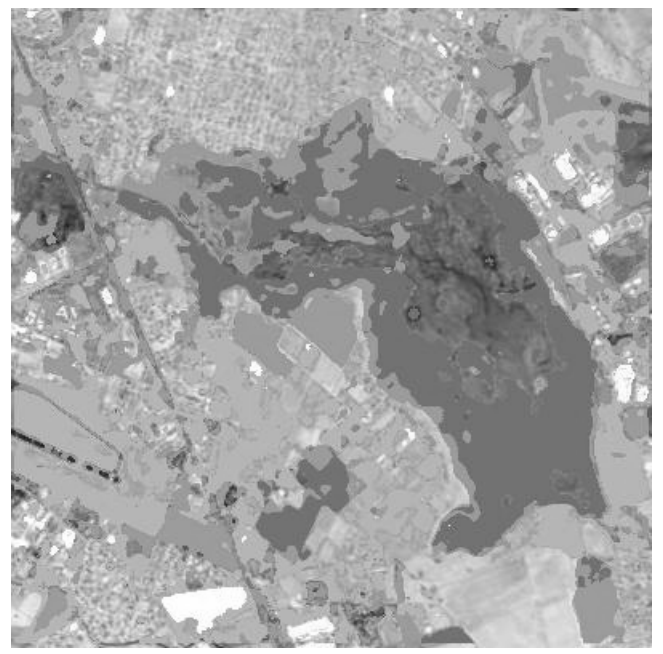

f. Dynamic prediction obtained with the MDF method for $\kappa=$ 41 discrete time.

Figure 3. Simulation results for dynamic MRSD map analysis. 


\section{CONCLUDING REMARKS}

From the presented simulations results, it is possible to deduce that the developed MDF method provides a possibility to perform the intelligent analysis of the dynamic behavior or the desired environmental map in both, spatial resolution and time evolution. This is achieved because the MDF algorithm aggregates the information of the MRSD collection of remote sensing images for a particular geographical region in discrete time and employs more detailed robust a priori information from the original reconstructed remote sensing scene. The resulting dynamic MRSD prediction map ensures a high accuracy in the estimation process and in the classification achieved.

A real-time process (RTP) can be defined as the study of software systems which are subject to a real-time constraint (i.e., operational deadlines from event to system response).

By contrast, a non-real-time system is one for which there is no deadline, even if fast response or high performance is desired or even preferred. The needs of RTP software are often addressed in the context of real-time operating systems, and synchronous programming languages, which provide frameworks on which to build RTP application software.

A RTP may be one where its application can be considered (within context) to be mission critical. Moreover, RTP can be said to have failed if they are not completed before their deadline, where their deadline is relative to an event. A deadline must be met, regardless of system load.

\section{FUTURE WORK}

The results reported on this paper shows the qualitative analysis of the overall performance of the MDF method applied to remote sensing data for geosimulation purposes. The application as an auxiliary tool in geophysical information retrieval and data interpretation for land use management and analysis, and a more extensive quantitative analysis of the results are a matter of further studies.

\section{ACKNOWLEDGEMENTS}

The author would like to thank the Instituto Tecnológico y de Estudios Superiores de Occidente (ITESO) of Mexico for the resources provided for this research under the project titled "Desarrollo de modelos adaptivos para el procesamiento digital de señales multiespectrales de percepción remota y su implementación como software de alto desempeño".

\section{REFERENCES}

Falkovich, S., Ponomaryov, V., and Shkvarko Y., 1989. Optimal Spatial-Temporal Signal Processing for Spread Radio Channels. Radio and Communication Press.
Fussell, J., Rundquist, D. and Harrington, J., 1986. On defining remote sensing. Journal of Photogrammetric Engineering \& Remote Sensing, Vol. 52, 1507-1511.

Grewal, M. and Andrews, A., 2001. Kalman Filtering: Theory and Practice using Matlab. John Wiley \& Sons.

Jensen, J., 2005. Introductory Digital Image Processing: A Remote Sensing Perspective, Prentice-Hall, U.S.A.

Johannsen, C., Petersen, G., Carter, P. and Morgan, M., 2003. Remote sensing: changing natural resource management. Journal of Soil \& Water Conservation, Vol. 58, 42-45.

Luenberger, D., 1979. Introduction to Dynamic Systems: Theory, Models and Applications. John Wiley \& Sons.

Marple, S., 1987. Digital Spectral Analysis. Prentice-Hall.

Mather, P., 2004. Computer Processing of Remotely-Sensed Images, John Wiley \& Sons, U.S.A.

Perry, S., Wong, H. and Guan, L., 2002. Adaptive Image Processing: A Computational Intelligence Perspective, CRC Press, U.S.A.

Shkvarko, Y., 2004a. Unifying Regularization and Bayesian Estimation Methods for Enhanced Imaging with Remotely Sensed Data. Part I - Theory. In IEEE Trans. Geoscience and Remote Sensing, pp. 923-931.

Shkvarko, Y., 2004b. Unifying Regularization and Bayesian Estimation Methods for Enhanced Imaging with Remotely Sensed Data. Part II - Implementation and Performance Issues. In IEEE Trans. Geoscience and Remote Sensing, pp. 932-940.

Shkvarko, Y., Villalon-Turrubiates, I., 2007. Remote Sensing Imagery and Signature Fields Reconstruction via Aggregation of Robust Regularization with Neural Computing. In Lecture Notes in Computer Sciences, Blanc-Talon J., Philips W., Popescu D., Scheunders P., Springer-Verlag, pp. 235-246.

Villalon-Turrubiates, I., 2014. Dynamical Characterization of Land-Use Classification using Multispectral Remote Sensing Data for Guadalajara Region. In Proceedings of the IEEE International Geoscience and Remote Sensing Symposium (IGARSS), pp. 145-148.

Yli-Harja, O., Astola, J. and Neuvo, Y., 1991. Analysis of the properties of median and weighted order median filters using threshold logic and stack filter representations. IEEE Transactions on Signal Processing, Vol. 39, 395-410. 